\title{
Mediatory role of inflammatory markers on the relationship between dietary energy density and body compositions among obese and overweight adult women: A cross-sectional study
}

\author{
khadijeh mirzaei ${ }^{1}$, niki bahrampour ${ }^{2}$, Atieh Mirzababaei ${ }^{1}$, and Farideh shiraseb ${ }^{2}$ \\ ${ }^{1}$ Tehran University of Medical Sciences \\ ${ }^{2}$ Affiliation not available
}

May 1, 2021

\begin{abstract}
Background: Obesity is a global issue. Energy density (ED) can influence on body compositions (BCs). Consumption of HED (high energy density) foods can increase body fat mass (BFM) and inflammatory markers. Methods: This study was a cross-sectional research among 391 women. Body composition analyzer (BIA) and food frequency questionnaire (FFQ) was used to assess BCs and food intake of individuals. Blood samples and serum level of high-sensitive C-reactive protein (hs-CRP), plasminogen activator inhibitor- 1 (PAI-1) and transforming growth factor- $\beta$ (TGF-beta) were collected. ED per one gram of foods were calculated and divided to quartiles. Linear logistic regression tests were used to investigate the association between BCs across quartiles of ED intake. Results: Results demonstrate skeletal muscle mass (SMM), total body water (TBW), intracellular water (ICW), fat free mass (FFM), visceral fat area (VFA) and fat free mass index (FFMI) was seem to be under the effect of hs-CRP among ED intakes. TBW, extracellular water (ECW), FFM with PAI-1, bone mineral content (BMC) with PAI-1 and TGF-beta, and Skeletal lean mass (SLM) with hs-CRP were inversely associated. Fat right arm (FRA), fat right arm (FLA), fat right and left leg (FRL,FLL), fat trunk, TBW, ICW, ECW, BFM, FFM, SMM, SLM, waist circumference (WC), FFMI and FMI were positively under the influence of TGF-beta after following higher ED food intakes. FRA, FLA, FRL, FLL, fat trunk, ICW, BFM, SMM, SLM, WC, FFMI and FMI were positively under the influence of PAI-1. Conclusion: All adipose tissue content of subcategories of BC were strongly associated with ED intake in the mediatory role of PAI-1 and TGF-beta.
\end{abstract}

\section{Hosted file}

article\selectlanguage\{english\}NB.pdf available at https://authorea.com/users/380090/ articles/520370-mediatory-role-of-inflammatory-markers-on-the-relationship-betweendietary-energy-density-and-body-compositions-among-obese-and-overweight-adult-women-across-sectional-study

\section{Hosted file}

result 5.pdf available at https://authorea.com/users/380090/articles/520370-mediatory-roleof-inflammatory-markers-on-the-relationship-between-dietary-energy-density-and-bodycompositions-among-obese-and-overweight-adult-women-a-cross-sectional-study 